Research Article

\title{
Multiple Dependent State Sampling-Based Chart Using Belief Statistic under Neutrosophic Statistics
}

\author{
Ahmed Ibrahim Shawky (D), Muhammad Aslam (D), and Khushnoor Khan (D) \\ Department of Statistics, Faculty of Science, King Abdulaziz University, Jeddah 21551, Saudi Arabia \\ Correspondence should be addressed to Muhammad Aslam; aslam_ravian@hotmail.com
}

Received 31 May 2020; Revised 26 July 2020; Accepted 1 August 2020; Published 27 August 2020

Academic Editor: Francisco Balibrea

Copyright (C) 2020 Ahmed Ibrahim Shawky et al. This is an open access article distributed under the Creative Commons Attribution License, which permits unrestricted use, distribution, and reproduction in any medium, provided the original work is properly cited.

In this paper, a control chart scheme has been introduced for the mean monitoring using gamma distribution for belief statistics using multiple dependent (deferred) state sampling under the neutrosophic statistics. The coefficients of the control chart and the neutrosophic average run lengths have been estimated for specific false alarm probabilities under various process conditions. The offered chart has been compared with the existing classical chart through simulation and the real data. From the comparison, it is concluded that the performance of the proposed chart is better than that of the existing chart in terms of average run length under uncertain environment. The proposed chart has the ability to detect a shift quickly than the existing chart. It has been observed that the proposed chart is efficient in quick monitoring of the out-of-control process and a cherished addition in the toolkit of the quality control personnel.

\section{Introduction}

The control chart is a key technique to statistical process control to ensure the quality of the production process. The technique of control chart requires the construction of a central line, and two control limits are known as the lower control limit (LCL) and upper control limit (UCL). The quality characteristic of interest is then plotted on this chart for the quick monitoring of an observation falling outside these two limits. The idea of control chart was floated by Shewhart A. Walter during the 1920s, and plenty of control chart techniques has been developed by researchers but remained unsuccessful to develop a robust control chart technique. Gamma distribution is the commonly employed probability distribution for estimating time between events in physical sciences. The gamma distribution is a well-fitted distribution to the failure between events and an excellent alternative to normal, log normal, and nonparametric approaches and routinely used in the control chart literature [1]. It has been concluded that the gamma distribution approaches to the normal distribution when the shape parameter is very large. Aslam et al. [2] proposed an MDS sampling chart using the transformation of the gamma to normal distribution. Aslam et al. [3] developed a control chart for the belief estimator using the interested quality characteristic which follows the gamma distribution. Zhang et al. [4] investigated the gamma chart for monitoring $r^{\text {th }}$ event in the time occurring events. Bhaumik and Gibbons [1] developed one-sided prediction intervals for environmental-based quality characteristics for gamma distribution. Al-Oraini and Rahim [5] developed an economic control chart using constraints to the optimization problem for gamma-distributed quality characteristics. Several research studies including [6-8] have used gamma distribution in developing control chart.

The technique of multiple dependent state (MDS) sampling was announced by Wortham and Baker [9] in which the decision for in-control process or out-of-control process is established upon not only the current information of the process but also considers the previous record of the process. Soundararajan and Vijayaraghavan [10] developed the acceptance sampling plan for the fixed number of units for fixed acceptance and limiting quality levels which involve the least sum of risks. Soundararajan and Vijayaraghavan 
[11] proposed a search procedure using conventional sampling plans for smaller sample sizes. Kuralmani and Govindaraju [12] presented the techniques and tables of MDS sampling plan for the selection of minimum sample size. The MDS sampling plans for the least sampling size were proposed by Govindaraju and Subramani [13] for indicated acceptable quality and limiting quality levels. Balamurali and Jun [14] suggested an MDS sampling plan for the normal distribution for variables. Aslam et al. [15] designed an MDS sampling control chart for average monitoring with two control limits. Aslam et al. [16] developed an MDS sampling plan for variable quality characteristics under process loss consideration. Aslam et al. [17] suggested an exponential distribution for the MDS sampling chart for a normal approximation using transformation. Aslam et al. [18] developed an attribute control chart for MDS sampling as compared to the traditional control charts. Balamurali et al. [19] designed an MDS sampling plan for the gamma-Poisson distribution using the Bayesian statistics. Yan et al. [20] proposed an MDS sampling plan based upon the coefficient of variation for the normally distributed quality characteristics. Aldosari et al. [21] developed an MDS sampling control chart for attribute quality characteristics using the repetitive group sampling scheme. Zhou et al. [22] adopted the MDS sampling scheme using a joint adaptive np chart for improved checking of the manufacturing process. Afshari and Sadeghpour Gildeh [23] described the MDS sampling plan for an attribute using the fuzzy environment. Aslam et al. developed an MDS sampling plan for the measurable quality characteristics by joining the features of the repetitive group sampling and MDS sampling for the process capability index.

There are many situations in the real world when the information may be determinate or indeterminate [24]. Such data are dealt with the theory of neutrosophic statistics introduced by Smarandache [25]. The neutrosophic statistics is defined as the generalization of conventional statistics (see [26]). During the last few years, the application of neutrosophic statistics has attracted the attention of various researchers due to its nice properties as the conventional statistics cannot be used when our data consist of vague, incomplete, uncertain, soggy, or unclear observations. Ye et al. [27] investigated different properties and measure the effect of indeterminate joint roughness coefficient values using neutrosophic numbers. Aslam [28] proposed a neutrosophic sampling plan for the loss consideration processes. Aslam and Al-Marshadi [29] developed a neutrosophic sampling plan for the regression estimator. Aslam and Arif [30] suggested a neutrosophic sampling plan for examining electrical devices. Aslam et al. [31] proposed a chart for reliability data under neutrosophic statistics. Aslam et al. [32] developed a control chart scheme for checking the inconsistency under the neutrosophic interval technique. Aslam et al. [33] developed an attribute control chart for the neutrosophic statistics. Jansi et al. [34] developed a correlation measure for the Pythagorean neutrosophic sets using the dependent neutrosophic components. Muralikrishna and Kumar [35] developed a neutrosophic technique for data relating to linear space. The application of neutrosophic statistics in the quality control literature can be seen in works including [34-43].

Aslam et al. [44] proposed the control chart for the gamma distribution using the belief statistic for the single sampling plan. By exploring the literature and best of our knowledge, there is no work on the control chart using the belief statistic for MDS. The process monitoring using the belief statistic for neutrosophic statistics under the gamma distribution using MDS sampling has not been studied by any researcher yet. In this paper, we will present the MDS control chart using the belief statistic under neutrosophic statistics. We expect that the proposed chart will perform better in neutrosophic average run length as compared to the previous chart in an uncertain environment. It is expected that the proposed chart will able to detect shifts earlier than the existing chart. In addition, it is expected that the proposed chart will ensure the quality of the product according to ISO 9001: 2015 Quality Management Systems (https://www.iso.org). The rest of the article is organized as follows: the scheme of the planned chart is given in Section 2. We described the simulation study of the planned chart in Section 3. Section 4 describes the advantages of the planned chart. A comparison of the planned chart with an existing control chart has been given in Section 5. Conclusions are described in the last section.

\section{Scheme of the Planned Chart}

Let $T_{N}$ be the neutrosophic time between events of the interested quality characteristic from the gamma distribution having cumulative distribution function as

$$
P\left(T_{N} \leq t_{N}\right)=1-\sum_{j=0}^{a_{N}-1} \frac{e^{-t_{N} / b}\left(t_{N} / b_{N}\right)^{j}}{j !},
$$

where $a_{\mathrm{N}}$ and $b_{\mathrm{N}}$ are the shape and scale parameters, respectively. Under the particular condition when $a_{N}=1$, then this distribution tends to form the exponential distribution. Wilson and Hilferty [45] described that if $T$ is distributed from the gamma distribution, then the transformation $T_{N}^{*}=T_{N}^{1 / 3}$ follows the normal distribution.

The random variable $T_{N}^{*}$ has the mean and variance as follows:

$$
\begin{aligned}
& \mu_{T_{N}^{*}}=E\left(T_{N}^{*}\right)=\frac{b_{N}^{1 / 3} \Gamma\left(a_{N}+1 / 3\right)}{\Gamma\left(a_{N}\right)} \\
& \sigma_{T_{N}^{*}}=b_{N}^{1 / 3} \sqrt{\frac{\Gamma\left(a_{N}+2 / 3\right)}{\Gamma\left(a_{N}\right)}-\left(\frac{\Gamma\left(a_{N}+1 / 3\right)}{\Gamma\left(a_{N}\right)}\right)^{2}} .
\end{aligned}
$$


Then, the approximately normal distribution of $T_{N}^{*}$ can be described as

$$
T_{N}^{*} \sim N\left(\frac{b_{N}^{1 / 3} \Gamma\left(a_{N}+1 / 3\right)}{\Gamma\left(a_{N}\right)}, b_{N}^{1 / 3}\left[\frac{\Gamma\left(a_{N}+2 / 3\right)}{\Gamma\left(a_{N}\right)}-\left(\frac{\Gamma\left(a_{N}+1 / 3\right)}{\Gamma\left(a_{N}\right)}\right)^{2}\right]\right)
$$

Data are collected for belief statistics with the assumption of a single observation $(n=1)$ of the targeted quality of interest. We assume that the $k^{\text {th }}$ observation be $T_{k}$ and $O_{k}=$ $\left(T_{1}, T_{2}, \ldots, T_{k}\right)$ for the $k^{\text {th }}$ iteration of the vector of observation. We further assume that $O_{k}=\left(T_{k}, O_{k-1}\right)$ and $B\left(O_{k}\right)$ are the posterior belief and the prior belief is $B\left(O_{k-1}\right)$. Here, our purpose is to select a new observation by updating $B\left(O_{k}\right)$ using $B\left(O_{k-1}\right)$. We required the updated posterior belief for the gamma distribution under the transformed variable $T_{N}^{*}=T_{N}^{1 / 3}$. For this purpose, the following equation is developed:

$$
\begin{aligned}
B\left(O_{k_{N}}\right) & =B\left(T_{k_{N}}, O_{k_{N}-1}\right) \\
& =\frac{B\left(O_{k_{N}-1}\right) e^{T_{N}^{*}-\mu_{T_{N}^{*}} / \sigma_{T_{N}^{*}}}}{B\left(O_{k_{N}-1}\right) e^{T_{N}^{*}-\mu_{T_{N}^{*}} / \sigma_{T_{N}^{*}}}+\left(1-B\left(O_{k_{N}-1}\right)\right)} .
\end{aligned}
$$

It is to be noted that the variable $T_{N}^{*}$ is without the subscript $k$.

Let the statistic proposed by Fallah Nezhad and Akhavan Niaki [46] as

$$
Z_{k_{N}}=\frac{B\left(O_{k_{N}}\right)}{1-B\left(O_{k_{N}}\right)}
$$

The above expression can be written as follows:

$$
Z_{k_{N}}=Z_{k_{N-1}} e^{T_{N}^{*}-\mu_{T_{N}^{*}} / \sigma_{T_{N}^{*}}}
$$

Let the specified starting value of $Z_{0}=1$ and $B\left(O_{0}\right)=0.5$ are being used, then the statistic given below follows normally distributed quality characteristic having zero mean and $k$ variance [46]. Then, the lower and upper control limits of the proposed control chart are given by

$$
\begin{aligned}
& \mathrm{UCL}_{N}=L_{1 N} \sqrt{k_{N}}, \\
& \operatorname{LCL}_{N}=L_{1 N} \sqrt{k_{N}}, \\
& \mathrm{UCL}_{N}=L_{2 N} \sqrt{k_{N}}, \\
& \operatorname{LCL}_{N}=L_{2 N} \sqrt{k_{N}},
\end{aligned}
$$

where $L$ denotes the chart coefficient to be estimated using the type I error and in-control average run lengths of the process.

The technique of the planned chart is elaborated in the subsequent steps as follows:

Step 1: choose an item randomly at the $k^{\text {th }}$ subgroup and calculate its quality characteristic $T_{k_{N}}$. Convert $T_{k_{N}}^{*}=T_{k_{N}}^{1 / 3}$ and then compute

$$
\ln \left(Z_{k_{N}}\right)=\ln \left(Z_{k_{N}}-1\right)+\frac{T_{k_{N}}^{*}-\mu_{T_{k_{N}}^{*}}}{\sigma_{T_{k_{N}}^{*}}}
$$

Step 2: if $\mathrm{LCL}_{N} \leq \ln \left(Z_{k_{N}}\right) \leq \mathrm{UCL}_{N}$, announce the process as the in-control process, and if $\ln \left(Z_{k_{N}}\right)>\mathrm{UCL}_{N}$ or $\ln \left(Z_{k_{N}}\right)>\mathrm{LCL}_{N}$, then the process is confirmed as out of control.

The measures of the planned chart for the in-control and the out-of-control process are computed with the assumption that we change the scale parameter of underlying distribution while the shape parameter remains fixed during the entire process. Let $b_{0 N}$ and $b_{1 N}$ denote the scale parameter of the incontrol and out-of-control process. The probability that the incontrol process is stated as the out-of-control process when actually process is in-control may be expressed as

$$
P_{i n 0 N}=P\left(\mathrm{LCL}_{N} \leq \operatorname{In}\left(Z_{k_{N}}\right) \leq \mathrm{UCL}_{N}\right) \text {. }
$$

After some simplification, equation (13) can be written as follows:

$$
P_{\text {in } 0 N}=2\left(\Phi\left(L_{2 N}\right)\right)-1 .
$$

Similarly, the probability for in-decision can be written as

$$
\begin{aligned}
P\left(\mathrm{LCL}_{N} \leq \ln \left(Z_{k_{N}}\right) \leq \mathrm{LCL}_{N}\right) \\
\quad+P\left(\mathrm{UCL}_{N} \leq \operatorname{In}\left(Z_{k_{N}}\right) \leq \mathrm{UCL}_{N}\right) \\
=2\left[\Phi_{N}\left(L_{1 N}\right)-\Phi_{N}\left(L_{2 N}\right)\right] .
\end{aligned}
$$

Here, it is worth noting that $P_{i n 0 N}$ does not involve $k_{N}$. The probability of in-control for the proposed chart is given by

$$
\begin{aligned}
P_{i n N}= & P_{i n 0 N}+\left[P\left(\mathrm{LCL}_{N} \leq \ln \left(Z_{k_{N}}\right) \leq \mathrm{LCL} 2_{N}\right)\right. \\
& \left.+P\left(\mathrm{UCL} 2_{N} \leq \ln \left(Z_{k_{N}}\right) \leq \mathrm{UCL}_{N}\right)\right] * P_{i n 0 N}^{m} .
\end{aligned}
$$

After some simplification, equation (16) can be written as follows:

$$
\begin{aligned}
P_{i n N}= & \left(2 \Phi_{N}\left(L_{2 N}\right)-1\right)+\left[2\left[\Phi_{N}\left(L_{1 N}\right)-\Phi_{N}\left(L_{2 N}\right)\right]\right] \\
& \times\left(2 \Phi_{N}\left(L_{2 N}\right)-1\right)^{m} .
\end{aligned}
$$


The evaluation of the functioning of the developed control chart is judged by calculating the average run length (ARL) that is very commonly suggested by the quality control researchers [47]. It is defined as the average of all the samples until the process shows the deterioration. The ARL of the in-control manner may be determined as follows:

$$
\mathrm{ARL}_{0}=\frac{1}{1-P_{\text {inN }}} .
$$

It is very common that no process operates smoothly for a long time without any alteration in the process. So the evaluation of the changed process provides us the effectiveness of the proposed scheme in haste and prompt indication of the out-of-control process. Let the scale factor of the gamma distribution has been moved from $b_{0}$ to $b_{1}=s b_{0}$ where $s$ is the amount of shift. Then, the average and the variance of the changing process are calculated as follows:

$$
\begin{aligned}
E\left(T_{N}^{*} \mid b_{1 N}\right) & =s^{1 / 3} b_{0 N}^{1 / 3} \frac{\Gamma\left(a_{N}+1 / 3\right)}{\Gamma\left(a_{N}\right)}, \\
\operatorname{Var}\left(T_{N}^{*} \mid b_{1 N}\right) & =s^{1 / 3} b_{0 N}^{2 / 3}\left[\frac{\Gamma\left(a_{N}+2 / 3\right)}{\Gamma\left(a_{N}\right)}-\left(\frac{\Gamma\left(a_{N}+1 / 3\right)}{\Gamma\left(a_{N}\right)}\right)^{2}\right] .
\end{aligned}
$$

Thus, the mean and variance of $\ln \left(Z_{k_{\mathrm{N}}}\right)$ at $\mathrm{b}_{1 \mathrm{~N}}$ which follows an approximately normal distribution are given as follows:

$$
\begin{aligned}
E\left[\ln \left(Z_{k_{N}}\right) \mid b_{1 n}\right) & =k_{N} \frac{\Gamma\left(a_{N}+1 / 3\right) / \Gamma(a)\left(s^{1 / 3}-1\right)}{\sqrt{\Gamma\left(a_{N}+2 / 3\right) / \Gamma\left(a_{N}\right)-\left(\Gamma\left(a_{N}+1 / 3\right) / \Gamma\left(a_{N}\right)\right)^{2}}}, \\
\operatorname{Var}\left(\ln \left(Z_{k_{N}}\right) \mid b_{1 n}\right) & =k_{N} s^{2 / 3} .
\end{aligned}
$$

So, the probability of the out-of-control process for the shifted process at $k^{\text {th }}$ sample is calculated as follows:

$$
\begin{aligned}
P_{\text {in } 1 N}= & P\left(\operatorname{LCL} 2_{N} \leq \ln \left(Z_{k_{N}}\right) \leq \mathrm{UCL} 2_{N}\right), \\
P_{\text {in } 1 N}= & P\left\{\ln \left(Z_{k_{N}}\right)<\mathrm{UCL} 2_{N} \mid b_{N}=b_{1 N}\right\}-P\left\{\ln \left(Z_{k_{N}}\right)<\mathrm{LCL} 2_{N} \mid b_{N}=b_{1 N}\right\}, \\
P_{\text {in } 1 N}= & P\left\{\ln \left(Z_{k_{N}}\right)<L 2_{N} \sqrt{k_{N}} \mid b_{N}=b_{1 N}\right\}-P\left\{\ln \left(Z_{k_{N}}\right)<-L 2_{N} \sqrt{k_{N}} \mid b_{N}=b_{1 N}\right\}, \\
P_{\text {in } 1 N}= & \Phi\left(\frac{L 2_{N} \sqrt{k_{N}}-\left(k_{N} \cdot \Gamma\left(a_{N}+1 / 3\right) / \Gamma\left(a_{N}\right)\left(s^{1 / 3}-1\right) / \sqrt{\Gamma\left(a_{N}+2 / 3\right) / \Gamma\left(a_{N}\right)-\left(\Gamma\left(a_{N}+1 / 3\right) / \Gamma\left(a_{N}\right)\right)^{2}}\right)}{\sqrt{k_{N} S^{2 / 3}}}\right) \\
& -\Phi\left(\frac{-L 2_{N} \sqrt{k_{N}}-\left(k_{N} \cdot \Gamma\left(a_{N}+1 / 3\right) / \Gamma\left(a_{N}\right)\left(s^{1 / 3}-1\right) / \sqrt{\Gamma\left(a_{N}+2 / 3\right) / \Gamma\left(a_{N}\right)-\left(\Gamma\left(a_{N}+1 / 3\right) / \Gamma\left(a_{N}\right)\right)^{2}}\right)}{\sqrt{k_{N} S^{2 / 3}}}\right) .
\end{aligned}
$$

The probability of in-decision for the shifted process is given by

$$
P\left(\operatorname{LCL}_{N} \leq \ln \left(Z_{k_{N}}\right) \leq \mathrm{LCL} 2_{N}\right)+P\left(\mathrm{UCL} 2_{N} \leq \ln \left(Z_{k_{N}}\right) \leq \mathrm{UCL}_{N}\right) .
$$


The simplified form of equation (26) is given by

$$
\begin{aligned}
= & \Phi\left(\frac{-L 2_{N} \sqrt{k_{N}}-k_{N} \cdot \Gamma\left(a_{N}+1 / 3\right) / \Gamma\left(a_{N}\right)\left(s^{1 / 3}-1\right) / \sqrt{\Gamma\left(a_{N}+2 / 3\right) / \Gamma\left(a_{N}\right)-\left(\Gamma\left(a_{N}+1 / 3\right) / \Gamma\left(a_{N}\right)\right)^{2}}}{\sqrt{k_{N} S^{2 / 3}}}\right) \\
& -\Phi\left(\frac{-L 1_{N} \sqrt{k_{N}}-k_{N} \cdot \Gamma\left(a_{N}+1 / 3\right) / \Gamma\left(a_{N}\right)\left(s^{1 / 3}-1\right) / \sqrt{\Gamma\left(a_{N}+2 / 3\right) / \Gamma\left(a_{N}\right)-\left(\Gamma\left(a_{N}+1 / 3\right) / \Gamma\left(a_{N}\right)\right)^{2}}}{\sqrt{k_{N} S^{2 / 3}}}\right)+ \\
& +\Phi\left(\frac{L 1_{N} \sqrt{k_{N}}-k_{N} \cdot \Gamma\left(a_{N}+1 / 3\right) / \Gamma\left(a_{N}\right)\left(s^{1 / 3}-1\right) / \sqrt{\Gamma\left(a_{N}+2 / 3\right) / \Gamma\left(a_{N}\right)-\left(\Gamma\left(a_{N}+1 / 3\right) / \Gamma\left(a_{N}\right)\right)^{2}}}{\sqrt{k_{N} S^{2 / 3}}}\right) \\
& -\Phi\left(\frac{L 2_{N} \sqrt{k_{N}}-k_{N} \cdot \Gamma\left(a_{N}+1 / 3\right) / \Gamma\left(a_{N}\right)\left(s^{1 / 3}-1\right) / \sqrt{\Gamma\left(a_{N}+2 / 3\right) / \Gamma\left(a_{N}\right)-\left(\Gamma\left(a_{N}+1 / 3\right) / \Gamma\left(a_{N}\right)\right)^{2}}}{\sqrt{k_{N} S^{2 / 3}}}\right)
\end{aligned}
$$

The probability of in-control for the shifted process is given by

$$
\begin{aligned}
& P_{1 \text { in } N} \mid m_{1 N}=\left(\Phi\left(\frac{\mathrm{L} 2_{N} \sqrt{\mathrm{k}_{N}}-\mathrm{k}_{N} \cdot \Gamma\left(a_{N}+1 / 3\right) / \Gamma\left(a_{N}\right)\left(\mathrm{s}^{1 / 3}-1\right) / \sqrt{\Gamma\left(a_{N}+2 / 3\right) / \Gamma\left(a_{N}\right)-\left(\Gamma\left(a_{N}+1 / 3\right) / \Gamma\left(a_{N}\right)\right)^{2}}}{\sqrt{\mathrm{k}_{N} s^{2 / 3}}}\right)\right. \\
& \left.-\Phi\left(\frac{-\mathrm{L} 2_{N} \sqrt{\mathrm{k}_{N}}-\mathrm{k}_{N} \cdot \Gamma\left(a_{N}+1 / 3\right) / \Gamma\left(a_{N}\right)\left(\mathrm{s}^{1 / 3}-1\right) / \sqrt{\Gamma\left(a_{N}+2 / 3\right) / \Gamma\left(a_{N}\right)-\left(\Gamma\left(a_{N}+1 / 3\right) / \Gamma\left(a_{N}\right)\right)^{2}}}{\sqrt{\mathrm{k}_{N} S^{2 / 3}}}\right)\right) \\
& \Phi\left(\frac{-\mathrm{L} 2_{N} \sqrt{\mathrm{k}_{N}}-\mathrm{k}_{N} \cdot \Gamma\left(a_{N}+1 / 3\right) / \Gamma\left(a_{N}\right)\left(\mathrm{s}^{1 / 3}-1\right) / \sqrt{\Gamma\left(a_{N}+2 / 3\right) / \Gamma\left(a_{N}\right)-\left(\Gamma\left(a_{N}+1 / 3\right) / \Gamma\left(a_{N}\right)\right)^{2}}}{\sqrt{\mathrm{k}_{N} s^{2 / 3}}}\right) \\
& -\Phi\left(\frac{-\mathrm{L} 1_{N} \sqrt{\mathrm{k}_{N}}-\mathrm{k}_{N} \cdot \Gamma\left(a_{N}+1 / 3\right) / \Gamma\left(a_{N}\right)\left(s^{1 / 3}-1\right) / \sqrt{\Gamma\left(a_{N}+2 / 3\right) / \Gamma\left(a_{N}\right)-\left(\Gamma\left(a_{N}+1 / 3\right) / \Gamma\left(a_{N}\right)\right)^{2}}}{\sqrt{\mathrm{k}_{N} s^{2 / 3}}}\right) \\
& + \\
& +\Phi\left(\frac{\mathrm{L} 1_{N} \sqrt{\mathrm{k}_{N}}-\mathrm{k}_{N} \cdot \Gamma\left(a_{N}+1 / 3\right) / \Gamma\left(a_{N}\right)\left(\mathrm{s}^{1 / 3}-1\right) / \sqrt{\Gamma\left(a_{N}+2 / 3\right) / \Gamma\left(a_{N}\right)-\left(\Gamma\left(a_{N}+1 / 3\right) / \Gamma\left(a_{N}\right)\right)^{2}}}{\sqrt{\mathrm{k}_{N} S^{2 / 3}}}\right) \\
& \left.-\Phi\left(\frac{\mathrm{L} 2_{N} \sqrt{\mathrm{k}_{N}}-\mathrm{k}_{N} \cdot \Gamma\left(a_{N}+1 / 3\right) / \Gamma\left(a_{N}\right)\left(\mathrm{s}^{1 / 3}-1\right) / \sqrt{\Gamma\left(a_{N}+2 / 3\right) / \Gamma\left(a_{N}\right)-\left(\Gamma\left(a_{N}+1 / 3\right) / \Gamma\left(a_{N}\right)\right)^{2}}}{\sqrt{\mathrm{k}_{N} S^{2 / 3}}}\right)\right] \\
& \times\left(\Phi\left(\frac{\mathrm{L} 2_{N} \sqrt{\mathrm{k}_{N}}-\mathrm{k}_{N} \cdot \Gamma\left(a_{N}+1 / 3\right) / \Gamma\left(a_{N}\right)\left(\mathrm{s}^{1 / 3}-1\right) / \sqrt{\Gamma\left(a_{N}+2 / 3\right) / \Gamma\left(a_{N}\right)-\left(\Gamma\left(a_{N}+1 / 3\right) / \Gamma\left(a_{N}\right)\right)^{2}}}{\sqrt{\mathrm{k}_{N} S^{2 / 3}}}\right)\right. \\
& \left.-\Phi\left(\frac{-\mathrm{L} 2_{N} \sqrt{\mathrm{k}_{N}}-\mathrm{k}_{N} \cdot \Gamma\left(a_{N}+1 / 3\right) / \Gamma\left(a_{N}\right)\left(s^{1 / 3}-1\right) / \sqrt{\Gamma\left(a_{N}+2 / 3\right) / \Gamma\left(a_{N}\right)-\left(\Gamma\left(a_{N}+1 / 3\right) / \Gamma\left(a_{N}\right)\right)^{2}}}{\sqrt{\mathrm{k}_{N} S^{2 / 3}}}\right)\right)^{m} \text {. }
\end{aligned}
$$


Similarly, the expression for the $(k+j)^{\text {th }}$ sample of the shifted process as the change occurs at $k-$ th sample is given as

$$
\begin{aligned}
P\{\mathrm{RL}=j\}= & \left(1-P_{\text {out }, k_{N}+1}^{1}\right)\left(1-P_{\text {out }, k_{N}+2}^{1}\right) \\
& \ldots\left(1-P_{\text {out }, k_{N}+j-1}^{1}\right) P_{\text {out }, k_{N}+j}^{1} .
\end{aligned}
$$

Here, $\mathrm{RL}$ is a run-length random variable indicating the out-of-control process.

So, the ARL of the shifted process is specified as follows:

$$
\begin{aligned}
\mathrm{ARL}_{1 N}= & P_{\text {out }, k_{N}+1}^{1}+2\left(1-P_{\text {out }, k_{N}+1}^{1}\right) P_{\text {out }, k_{N}+2}^{1} \\
& +3\left(1-P_{\text {out }, k_{N}+1}^{1}\right)\left(1-P_{\text {out }, k_{N}+2}^{1}\right) P_{\text {out }, k_{N}+3}^{1}+\cdots
\end{aligned}
$$

Suppose $r_{0}$ be the in-control specified ARL. Then, the procedure to compute the control chart coefficient and ARL of the shifted process may be followed as

Step 1: select a series of control chart coefficient $L_{N}$

Step 2: compute $L_{N}$ such that $\mathrm{ARL}_{0 N} \geq r_{0}$

Step 3: for a constant value of $k_{N}$ and many values of shifts $s$, we calculate $P_{\text {out, } k_{N}}^{1}$ using equation (18)

Step 4: compute $\mathrm{ARL}_{1 N}$ for fixed $k_{N}$ and various shifted values of $s$.

The values of NARL for various parameters are presented in Tables 1-4. From Tables 1-4, it can be witnessed that NARL decreases as $k$ increases from $k \epsilon[3,5]$ to $k \epsilon$ $[8,10]$. For example, from Table 1 , the values of NARL are $[121.05,100.93],[62.51,40.35]$, and $[33.11,17.9]$ for the shifts, $s=1.10,1.20$, and 1.30 and when $k \epsilon[3,5]$ and when $k \in[8,10]$ from Table 2 , then the values of NARL are [90.67, 76.19], [31.95, 21.57], and [13.29, 8.13] for $r_{0}=200$. We also observe that as $a$ decreases from $a \in[1.95,2.05]$ to $a \epsilon[0.95,1.05]$, the values of NARL increase.

\section{Comparison of the Planned Chart with the Existing Chart}

In this section, the benefits of the neutrosophic control chart under MDS sampling for the belief statistics will be discussed. Aslam et al. [44] proposed the control chart for belief statistics using the single sampling when the observations collected are vague, incomplete, unclear, imprecise, or uncertain and showed that it was an effective and efficient chart as compared to chart under classical statistics. According to $[48,49]$, a control chart having the smaller values of ARL is said to be a more efficient chart. In this section, we will compare the proposed chart with Aslam et al. [44].

3.1. Compression in NARL. We will compare the planned neutrosophic chart using MDS sampling for the belief statistics with the existing neutrosophic chart for belief statistics provided by Aslam et al. [44]. As described earlier,
TABle 1: The values of NARL when $a=1.95$ and 2.05 and $k=3$ and 5 .

\begin{tabular}{lccc}
\hline \multicolumn{4}{c}{$a \epsilon[1.95,2.05] ; k \epsilon[3,5]$} \\
\hline$k_{1 N}$ & {$[3.2104,3.2996]$} & {$[3.4042,3.4512]$} & {$[3.1128,3.2105]$} \\
$K_{2 N}$ & {$[2.0148,2.1321]$} & {$[2.0843,2.2099]$} & {$[2.2992,2.337]$} \\
\hline Shift $(s)$ & & ARL $_{N}$ \\
\hline 4.00 & {$[1.11,1.02]$} & {$[1.12,1.02]$} & {$[1.16,1.03]$} \\
3.00 & {$[1.34,1.10]$} & {$[1.38,1.11]$} & {$[1.47,1.13]$} \\
2.80 & {$[1.45,1.14]$} & {$[1.50,1.16]$} & {$[1.62,1.19]$} \\
2.50 & {$[1.71,1.25]$} & {$[1.80,1.28]$} & {$[2.00,1.33]$} \\
2.25 & {$[2.13,1.42]$} & {$[2.29,1.47]$} & {$[2.60,1.55]$} \\
2.00 & {$[2.99,1.76]$} & {$[3.31,1.85]$} & {$[3.85,2.01]$} \\
1.90 & {$[3.60,2.01]$} & {$[4.05,2.13]$} & {$[4.75,2.34]$} \\
1.80 & {$[4.49,2.37]$} & {$[5.14,2.55]$} & {$[6.10,2.84]$} \\
1.70 & {$[5.86,2.94]$} & {$[6.86,3.23]$} & {$[8.20,3.66]$} \\
1.60 & {$[8.09,3.91]$} & {$[9.69,4.41]$} & {$[11.68,5.07]$} \\
1.50 & {$[11.93,5.7]$} & {$[14.71,6.63]$} & {$[17.81,7.78]$} \\
1.40 & {$[19.03,9.37]$} & {$[24.26,11.34]$} & {$[29.38,13.51]$} \\
1.30 & {$[33.11,17.9]$} & {$[43.88,22.69]$} & {$[53.00,27.34]$} \\
1.20 & {$[62.51,40.35]$} & {$[86.59,54.10]$} & {$[104.3,65.43]$} \\
1.10 & {$[121.05,100.93]$} & {$[175.52,144.23]$} & {$[213,175.21]$} \\
1.00 & {$[200.56,200.33]$} & {$[301.11,301.00]$} & {$[371.94,371.38]$} \\
0.80 & {$[137.4,72.63]$} & {$[201.93,103.4]$} & {$[264.41,134.97]$} \\
0.75 & {$[96.92,41.99]$} & {$[139.73,58.2]$} & {$[192.24,78.17]$} \\
0.70 & {$[64.90,23.81]$} & {$[91.62,32.07]$} & {$[133.4,43.87]$} \\
0.60 & {$[26.44,7.87]$} & {$[35.66,9.91]$} & {$[57.36,13.44]$} \\
0.50 & {$[10.07,3.05]$} & {$[12.88,3.53]$} & {$[21.57,4.47]$} \\
0.40 & {$[3.86,1.57]$} & {$[4.62,1.69]$} & {$[7.36,1.92]$} \\
0.30 & {$[1.71,1.10]$} & {$[1.88,1.13]$} & {$[2.57,1.18]$} \\
0.25 & {$[1.29,1.02]$} & {$[1.36,1.03]$} & {$[1.66,1.05]$} \\
0.15 & {$[1.01,1.00]$} & {$[1.02,1.00]$} & {$[1.04,1.00]$} \\
0.10 & {$[1,1]$} & {$[1,1]$} & {$[1,1]$} \\
0.05 & {$[1,1]$} & {$[1,1]$} & {$[1,1]$} \\
\hline & & \multicolumn{3}{c}{ ] $]$} \\
\hline
\end{tabular}

NARL is used for examining the efficiency of any chart for the speedy and rapid indication of the out-of-control situation. From Table 5 by matching the ARLs of the planned chart with the existing Aslam et al. [44] chart, it can be witnessed that ARL measures of the planned control chart are lesser than the NARL values of the existing chart for all process shifts, $s$. For example, for a shift of 0.75 , the existing chart is expected to detect the shift from $116^{\text {th }}$ to $218^{\text {th }}$ sample. On the other hand, the proposed chart will detect the shift from $78^{\text {th }}$ to $192^{\text {nd }}$ sample. From this study, it is concluded that the proposed chart has the ability to detect a shift earlier than the existing chart. Therefore, the use of the proposed control chart in the industry will help to improve the quality of the product. This finding is the same as mentioned in ISO 9001: 2015 Quality Management Systems (https://www.iso.org).

3.2. Comparison by Simulation Study. The comparison of the planned chart with the existing chart has also been presented by simulation data given in Tables 6 and 7. Thus, the first 20 observations are created from the in-control process having $k_{N}=[3,5], a_{N}=[1.95,2.05]$, and $b_{N}=[2,2.2]$ and the next 
TABLE 2: The values of NARL when $a=1.95$ and 2.05 and $k=8$ and 10 .

\begin{tabular}{|c|c|c|c|}
\hline & \multicolumn{3}{|c|}{$a \in[1.95,2.05] ; k \in[8,10]$} \\
\hline$k_{1 N}$ & {$[3.022,3.7678]$} & {$[3.6019,4.2229]$} & {$[3.3592,3.6508]$} \\
\hline$K_{2 N}$ & {$[2.0893,2.1015]$} & {$[2.0616,2.1804]$} & {$[2.1514,2.2392]$} \\
\hline Shift $(s)$ & \multicolumn{3}{|c|}{$\mathrm{ARL}_{N}$} \\
\hline 4.00 & {$[1,1]$} & {$[1,1]$} & {$[1,1]$} \\
\hline 3.00 & {$[1.02,1.01]$} & {$[1.02,1.01]$} & {$[1.03,1.01]$} \\
\hline 2.80 & {$[1.04,1.01]$} & {$[1.04,1.01]$} & {$[1.04,1.02]$} \\
\hline 2.50 & {$[1.08,1.03]$} & {$[1.08,1.04]$} & {$[1.09,1.04]$} \\
\hline 2.25 & {$[1.17,1.08]$} & {$[1.17,1.09]$} & {$[1.19,1.1]$} \\
\hline 2.00 & {$[1.37,1.19]$} & {$[1.38,1.21]$} & {$[1.42,1.23]$} \\
\hline 1.90 & {$[1.52,1.27]$} & {$[1.54,1.31]$} & {$[1.6,1.33]$} \\
\hline 1.80 & {$[1.76,1.40]$} & {$[1.8,1.45]$} & {$[1.88,1.49]$} \\
\hline 1.70 & {$[2.14,1.60]$} & {$[2.22,1.68]$} & {$[2.35,1.74]$} \\
\hline 1.60 & {$[2.82,1.96]$} & {$[2.98,2.1]$} & {$[3.19,2.18]$} \\
\hline 1.50 & {$[4.08,2.64]$} & {$[4.47,2.91]$} & {$[4.84,3.06]$} \\
\hline 1.40 & {$[6.77,4.14]$} & {$[7.74,4.76]$} & {$[8.5,5.09]$} \\
\hline 1.30 & {$[13.29,8.13]$} & {$[16.17,9.89]$} & {$[18.04,10.8]$} \\
\hline 1.20 & {$[31.95,21.57]$} & {$[42.16,28.11]$} & {$[47.94,31.51]$} \\
\hline 1.10 & {$[90.67,76.19]$} & {$[130.59,107.63]$} & {$[153.49,125.65]$} \\
\hline 1.00 & {$[201.15,202.12]$} & {$[301.9,303.82]$} & {$[373.1,374.48]$} \\
\hline 0.80 & {$[47.94,26.73]$} & {$[61.2,35.94]$} & {$[75.35,42.64]$} \\
\hline 0.75 & {$[25.69,12.79]$} & {$[30.64,16.47]$} & {$[37.74,19.25]$} \\
\hline 0.70 & {$[13.77,6.55]$} & {$[15.52,8.06]$} & {$[18.97,9.22]$} \\
\hline 0.60 & {$[4.34,2.34]$} & {$[4.53,2.62]$} & {$[5.31,2.84]$} \\
\hline 0.50 & {$[1.81,1.31]$} & {$[1.82,1.37]$} & {$[1.99,1.43]$} \\
\hline 0.40 & {$[1.14,1.04]$} & {$[1.13,1.05]$} & {$[1.17,1.06]$} \\
\hline 0.30 & {$[1.01,1]$} & {$[1.01,1]$} & {$[1.01,1]$} \\
\hline 0.25 & {$[1,1]$} & {$[1,1]$} & {$[1,1]$} \\
\hline 0.15 & {$[1,1]$} & {$[1,1]$} & {$[1,1]$} \\
\hline 0.10 & {$[1,1]$} & {$[1,1]$} & {$[1,1]$} \\
\hline 0.05 & {$[1,1]$} & {$[1,1]$} & {$[1,1]$} \\
\hline
\end{tabular}

TABLE 3: The values of NARL when $a=0.95$ and 1.05 and $k=3$ and 5 .

\begin{tabular}{|c|c|c|c|}
\hline & \multicolumn{3}{|c|}{$a \in[0.95,1.05] ; k \in[3,5]$} \\
\hline$k_{1 N}$ & {$[3.4983,3.6534]$} & {$[3.449,3.8141]$} & {$[3.6313,4.0783]$} \\
\hline$K_{2 N}$ & {$[1.9746,2.1033]$} & {$[2.0774,2.185]$} & {$[2.1067,2.2228]$} \\
\hline Shift $(s)$ & \multicolumn{3}{|c|}{$\mathrm{ARL}_{N}$} \\
\hline 4.00 & {$[1.55,1.18]$} & {$[1.62,1.2]$} & {$[1.65,1.21]$} \\
\hline 3.00 & {$[2.27,1.47]$} & {$[2.44,1.52]$} & {$[2.54,1.54]$} \\
\hline 2.80 & {$[2.59,1.59]$} & {$[2.81,1.66]$} & {$[2.95,1.69]$} \\
\hline 2.50 & {$[3.36,1.89]$} & {$[3.70,1.99]$} & {$[3.94,2.05]$} \\
\hline 2.25 & {$[4.50,2.34]$} & {$[5.06,2.52]$} & {$[5.46,2.63]$} \\
\hline 2.00 & {$[6.69,3.25]$} & {$[7.73,3.6]$} & {$[8.49,3.82]$} \\
\hline 1.90 & {$[8.16,3.88]$} & {$[9.54,4.37]$} & {$[10.57,4.68]$} \\
\hline 1.80 & {$[10.22,4.82]$} & {$[12.13,5.53]$} & {$[13.56,5.98]$} \\
\hline 1.70 & {$[13.21,6.26]$} & {$[15.95,7.33]$} & {$[18.02,8.02]$} \\
\hline 1.60 & {$[17.71,8.59]$} & {$[21.81,10.32]$} & {$[24.93,11.45]$} \\
\hline 1.50 & {$[24.75,12.62]$} & {$[31.16,15.58]$} & {$[36.08,17.56]$} \\
\hline 1.40 & {$[36.14,20.02]$} & {$[46.75,25.56]$} & {$[54.85,29.32]$} \\
\hline 1.30 & {$[55.12,34.6]$} & {$[73.6,45.9]$} & {$[87.6,53.65]$} \\
\hline 1.20 & {$[86.83,64.46]$} & {$[120.38,89.28]$} & {$[145.3,106.53]$} \\
\hline 1.10 & {$[136.74,122.13]$} & {$[197.6,177.02]$} & {$[241.52,215.44]$} \\
\hline 1.00 & {$[200.78,200.53]$} & {$[301.32,301.36]$} & {$[371.79,372.22]$} \\
\hline 0.80 & {$[235.2,154.47]$} & {$[362.15,229.88]$} & {$[447.56,281.83]$} \\
\hline 0.75 & {$[206.85,111.91]$} & {$[318.34,164.28]$} & {$[391.37,199.76]$} \\
\hline
\end{tabular}


TABLE 3: Continued.

\begin{tabular}{lccc}
\hline \multicolumn{3}{c}{$a \epsilon[0.95,1.05] ; k \epsilon[3,5]$} & \\
\hline 0.70 & {$[171.25,76.55]$} & {$[263.51,110.72]$} & {$[321.6,133.47]$} \\
0.60 & {$[101.35,32.33]$} & {$[156.15,45.21]$} & {$[187.25,53.49]$} \\
0.50 & {$[51.53,12.72]$} & {$[79.21,17]$} & {$[93.29,19.66]$} \\
0.40 & {$[22.91,4.98]$} & {$[34.67,6.23]$} & {$[40.1,6.98]$} \\
0.30 & {$[8.84,2.17]$} & {$[12.8,2.49]$} & {$[14.5,2.67]$} \\
0.25 & {$[5.21,1.57]$} & {$[7.26,1.72]$} & {$[8.1,1.8]$} \\
0.15 & {$[1.78,1.06]$} & {$[1.15,1.08]$} & {$[2.29,1.09]$} \\
0.10 & {$[1.18,1]$} & {$[1.01,1]$} & {$[1.32,1.01]$} \\
0.05 & {$[1.01,1]$} & {$[1.01,1]$} \\
\hline
\end{tabular}

TABLE 4: The values of NARL when $a=0.95$ and 1.05 and $k=8$ and 10 .

\begin{tabular}{|c|c|c|c|}
\hline & \multicolumn{3}{|c|}{$a \in[0.95,1.05] ; k \in[8,10]$} \\
\hline$k_{1 N}$ & {$[3.1112,3.779]$} & {$[3.7071,4.0566]$} & {$[3.522,3.7427]$} \\
\hline$K_{2 N}$ & {$[2.0442,2.1009]$} & {$[2.0576,2.1815]$} & {$[2.1181,2.2313]$} \\
\hline Shift $(s)$ & \multicolumn{3}{|c|}{$\mathrm{ARL}_{N}$} \\
\hline 4.00 & {$[1.07,1.02]$} & {$[1.07,1.03]$} & {$[1.08,1.03]$} \\
\hline 3.00 & {$[1.24,1.11]$} & {$[1.25,1.12]$} & {$[1.27,1.13]$} \\
\hline 2.80 & {$[1.32,1.15]$} & {$[1.34,1.17]$} & {$[1.36,1.18]$} \\
\hline 2.50 & {$[1.52,1.27]$} & {$[1.57,1.3]$} & {$[1.61,1.32]$} \\
\hline 2.25 & {$[1.85,1.44]$} & {$[1.95,1.49]$} & {$[2.01,1.53]$} \\
\hline 2.00 & {$[2.53,1.8]$} & {$[2.75,1.9]$} & {$[2.86,1.96]$} \\
\hline 1.90 & {$[3.02,2.05]$} & {$[3.34,2.2]$} & {$[3.49,2.28]$} \\
\hline 1.80 & {$[3.73,2.44]$} & {$[4.23,2.65]$} & {$[4.45,2.76]$} \\
\hline 1.70 & {$[4.85,3.04]$} & {$[5.64,3.38]$} & {$[5.97,3.55]$} \\
\hline 1.60 & {$[6.68,4.07]$} & {$[8.02,4.65]$} & {$[8.56,4.93]$} \\
\hline 1.50 & {$[9.9,5.99]$} & {$[12.32,7.05]$} & {$[13.29,7.57]$} \\
\hline 1.40 & {$[16,9.91]$} & {$[20.8,12.14]$} & {$[22.71,13.23]$} \\
\hline 1.30 & {$[28.58,19.01]$} & {$[39.04,24.38]$} & {$[43.33,27.09]$} \\
\hline 1.20 & {$[56.37,42.68]$} & {$[81.05,57.77]$} & {$[92.08,65.84]$} \\
\hline 1.10 & {$[115.95,104.55]$} & {$[173.78,150.2]$} & {$[204.15,177.31]$} \\
\hline 1.00 & {$[200.13,201.81]$} & {$[305.58,303.31]$} & {$[371.16,370.54]$} \\
\hline 0.80 & {$[117.09,75.62]$} & {$[168.59,107.95]$} & {$[204.29,129.77]$} \\
\hline 0.75 & {$[78.76,43.72]$} & {$[108.53,60.82]$} & {$[131.47,72.62]$} \\
\hline 0.70 & {$[50.62,24.77]$} & {$[66.61,33.53]$} & {$[80.64,39.68]$} \\
\hline 0.60 & {$[19.34,8.19]$} & {$[23.42,10.38]$} & {$[28.09,11.95]$} \\
\hline 0.50 & {$[7.13,3.15]$} & {$[8.07,3.69]$} & {$[9.44,4.08]$} \\
\hline 0.40 & {$[2.80,1.60]$} & {$[3.00,1.74]$} & {$[3.36,1.83]$} \\
\hline 0.30 & {$[1.40,1.11]$} & {$[1.43,1.14]$} & {$[1.51,1.16]$} \\
\hline 0.25 & {$[1.14,1.03]$} & {$[1.15,1.04]$} & {$[1.18,1.05]$} \\
\hline 0.15 & {$[1,1]$} & {$[1,1]$} & {$[1,1]$} \\
\hline 0.10 & {$[1,1]$} & {$[1,1]$} & {$[1,1]$} \\
\hline 0.05 & {$[1,1]$} & {$[1,1]$} & {$[1,1]$} \\
\hline
\end{tabular}

20 observations are created from the deteriorated process having $s^{*} b_{N}=[2,2.2]$ where $s=1.40$. Figure 1 shows the proposed chart, Figure 2 shows the Aslam et al. [44] chart, and Figure 3 shows the Shewhart chart under the classical statistics. By comparing these figures, it can be seen that the proposed chart detects shift near to $33^{\text {rd }}$ sample. On the other hand, Figures 2 and 3 do not show any shift in the process. Figures 1-3 have been given for simulated data which show better detecting ability of the indeterminacy data of the proposed chart. From the simulation study, it is concluded that the proposed chart is better in detecting the shift in the process while the existing chart does not detect the shift. Therefore, the use of the proposed chart will minimize nonconforming items.

\section{Application of the Proposed Chart in the Healthcare Department}

In this section, we will discuss the application of the proposed chart using the Urinary Tract Infection (UTI) data 
TABLE 5: The NARL values of the proposed and the existing charts when $a \epsilon[1.95,2.05]$ and $k=3,5$.

\begin{tabular}{|c|c|c|c|c|c|c|}
\hline \multirow{4}{*}{$\begin{array}{l}k_{N} \\
k_{1 N} \\
K_{2 N}\end{array}$} & \multicolumn{6}{|c|}{$a \in[1.95,2.05] ; k \in[3,5]$} \\
\hline & Existing & Proposed & Existing & Proposed & Existing & Proposed \\
\hline & {$[2.8071,2.8141]$} & {$[3.2104,3.2996]$} & {$[2.9354,2.9416]$} & {$[3.4042,3.4512]$} & {$[3.0003,3.0012]$} & {$[3.1128,3.2105]$} \\
\hline & & {$[2.0148,2.1321]$} & & {$[2.0843,2.2099]$} & & {$[2.2992,2.337]$} \\
\hline Shift $(s)$ & \multicolumn{6}{|c|}{$\mathrm{ARL}_{N}$} \\
\hline 4.00 & {$[1.28,1.06]$} & {$[1.11,1.02]$} & {$[1.32,1.07]$} & {$[1.12,1.02]$} & {$[1.34,1.07]$} & {$[1.16,1.03]$} \\
\hline 3.00 & {$[1.76,1.24]$} & {$[1.34,1.10]$} & {$[1.88,1.28]$} & {$[1.38,1.11]$} & {$[1.95,1.31]$} & {$[1.47,1.13]$} \\
\hline 2.80 & {$[1.98,1.34]$} & {$[1.45,1.14]$} & {$[2.13,1.4]$} & {$[1.50,1.16]$} & {$[2.22,1.43]$} & {$[1.62,1.19]$} \\
\hline 2.50 & {$[2.5,1.58]$} & {$[1.71,1.25]$} & {$[2.75,1.68]$} & {$[1.80,1.28]$} & {$[2.89,1.73]$} & {$[2.00,1.33]$} \\
\hline 2.25 & {$[3.27,1.97]$} & {$[2.13,1.42]$} & {$[3.67,2.13]$} & {$[2.29,1.47]$} & {$[3.91,2.22]$} & {$[2.60,1.55]$} \\
\hline 2.00 & {$[4.75,2.74]$} & {$[2.99,1.76]$} & {$[5.48,3.05]$} & {$[3.31,1.85]$} & {$[5.92,3.22]$} & {$[3.85,2.01]$} \\
\hline 1.90 & {$[5.74,3.28]$} & {$[3.60,2.01]$} & {$[6.71,3.7]$} & {$[4.05,2.13]$} & {$[7.29,3.93]$} & {$[4.75,2.34]$} \\
\hline 1.80 & {$[7.13,4.05]$} & {$[4.49,2.37]$} & {$[8.47,4.66]$} & {$[5.14,2.55]$} & {$[9.28,4.98]$} & {$[6.10,2.84]$} \\
\hline 1.70 & {$[9.18,5.24]$} & {$[5.86,2.94]$} & {$[11.1,6.12]$} & {$[6.86,3.23]$} & {$[12.26,6.61]$} & {$[8.20,3.66]$} \\
\hline 1.60 & {$[12.32,7.12]$} & {$[8.09,3.91]$} & {$[15.19,8.51]$} & {$[9.69,4.41]$} & {$[16.96,9.28]$} & {$[11.68,5.07]$} \\
\hline 1.50 & {$[17.4,10.34]$} & {$[11.93,5.7]$} & {$[21.93,12.67]$} & {$[14.71,6.63]$} & {$[24.76,13.98]$} & {$[17.81,7.78]$} \\
\hline 1.40 & {$[26.08,16.27]$} & {$[19.03,9.37]$} & {$[33.74,20.51]$} & {$[24.26,11.34]$} & {$[38.61,22.94]$} & {$[29.38,13.51]$} \\
\hline 1.30 & {$[41.89,28.25]$} & {$[33.11,17.9]$} & {$[55.90,36.86]$} & {$[43.88,22.69]$} & {$[64.99,41.90]$} & {$[53.00,27.34]$} \\
\hline 1.20 & {$[72.12,54.92]$} & {$[62.51,40.35]$} & {$[99.88,74.68]$} & {$[86.59,54.10]$} & {$[118.35,86.58]$} & {$[104.3,65.43]$} \\
\hline 1.10 & {$[127.96,115.71]$} & {$[121.05,100.93]$} & {$[185.00,165.6]$} & {$[175.52,144.23]$} & {$[224.15,196.69]$} & {$[213,175.21]$} \\
\hline 1.00 & {$[200.02,204.41]$} & {$[200.56,200.33]$} & {$[300.17,306.26]$} & {$[301.11,301.00]$} & {$[370.82,371.83]$} & {$[371.94,371.38]$} \\
\hline 0.80 & {$[152.58,98.67]$} & {$[137.4,72.63]$} & {$[227.95,143.72]$} & {$[201.93,103.4]$} & {$[281.15,172.31]$} & {$[264.41,134.97]$} \\
\hline 0.75 & {$[119.34,67.59]$} & {$[96.92,41.99]$} & {$[177.29,97.32]$} & {$[139.73,58.2]$} & {$[218.11,116.09]$} & {$[192.24,78.17]$} \\
\hline 0.70 & {$[90.73,45.44]$} & {$[64.90,23.81]$} & {$[134.07,64.61]$} & {$[91.62,32.07]$} & {$[164.56,76.64]$} & {$[133.4,43.87]$} \\
\hline 0.60 & {$[49.26,19.71]$} & {$[26.44,7.87]$} & {$[71.88,27.17]$} & {$[35.66,9.91]$} & {$[87.73,31.78]$} & {$[57.36,13.44]$} \\
\hline 0.50 & {$[24.63,8.22]$} & {$[10.07,3.05]$} & {$[35.26,10.86]$} & {$[12.88,3.53]$} & {$[42.65,12.45]$} & {$[21.57,4.47]$} \\
\hline 0.40 & {$[11.21,3.44]$} & {$[3.86,1.57]$} & {$[15.56,4.26]$} & {$[4.62,1.69]$} & {$[18.55,4.75]$} & {$[7.36,1.92]$} \\
\hline 0.30 & {$[4.64,1.6]$} & {$[1.71,1.10]$} & {$[6.1,1.82]$} & {$[1.88,1.13]$} & {$[7.09,1.95]$} & {$[2.57,1.18]$} \\
\hline 0.25 & {$[2.91,1.22]$} & {$[1.29,1.02]$} & {$[3.68,1.32]$} & {$[1.36,1.03]$} & {$[4.19,1.37]$} & {$[1.66,1.05]$} \\
\hline 0.15 & {$[1.27,1]$} & {$[1.01,1.00]$} & {$[1.4,1.01]$} & {$[1.02,1.00]$} & {$[1.49,1.01]$} & {$[1.04,1.00]$} \\
\hline 0.10 & {$[1.03,1]$} & {$[1,1]$} & {$[1.05,1]$} & {$[1,1]$} & {$[1.07,1]$} & {$[1,1]$} \\
\hline 0.05 & {$[1,1]$} & {$[1,1]$} & {$[1,1]$} & {$[1,1]$} & {$[1,1]$} & {$[1,1]$} \\
\hline
\end{tabular}

TABLE 6: The simulated data for the proposed control chart.

\begin{tabular}{|c|c|c|c|}
\hline Sr. no. & $B(k)$ & $z(k)$ & $\ln \left(Z_{k_{N}}\right)$ \\
\hline 1 & {$[0.007,0.569]$} & {$[0.007,1.323]$} & {$[-5.016,0.28]$} \\
\hline 2 & {$[0.374,0.246]$} & {$[0.598,0.326]$} & {$[-0.515,-1.122]$} \\
\hline 3 & {$[0.308,0.888]$} & {$[0.445,7.959]$} & {$[-0.811,2.074]$} \\
\hline 4 & {$[0.702,0.991]$} & {$[2.359,105.995]$} & {$[0.858,4.663]$} \\
\hline 5 & {$[0.454,0.006]$} & {$[0.832,0.006]$} & {$[-0.184,-5.084]$} \\
\hline 6 & {$[0.744,0.447]$} & {$[2.906,0.808]$} & {$[1.067,-0.214]$} \\
\hline 7 & {$[0.64,0.269]$} & {$[1.781,0.368]$} & {$[0.577,-1.001]$} \\
\hline 8 & {$[0.035,0.972]$} & {$[0.036,34.967]$} & {$[-3.327,3.554]$} \\
\hline 9 & {$[0.896,0.565]$} & {$[8.659,1.3]$} & {$[2.159,0.263]$} \\
\hline 10 & {$[0.155,0.493]$} & {$[0.183,0.973]$} & {$[-1.698,-0.027]$} \\
\hline 11 & {$[0.273,0.882]$} & {$[0.375,7.501]$} & {$[-0.981,2.015]$} \\
\hline 12 & {$[0.917,0.727]$} & {$[11.026,2.656]$} & {$[2.4,0.977]$} \\
\hline 13 & {$[0.499,0.964]$} & {$[0.997,26.938]$} & {$[-0.003,3.294]$} \\
\hline 14 & {$[0.732,0.818]$} & {$[2.735,4.492]$} & {$[1.006,1.502]$} \\
\hline 15 & {$[0.023,0.261]$} & {$[0.023,0.353]$} & {$[-3.754,-1.041]$} \\
\hline 16 & {$[0.465,0.988]$} & {$[0.868,79.71]$} & {$[-0.141,4.378]$} \\
\hline 17 & {$[0.146,0.841]$} & {$[0.171,5.303]$} & {$[-1.768,1.668]$} \\
\hline 18 & {$[0.873,0.972]$} & {$[6.844,34.4]$} & {$[1.923,3.538]$} \\
\hline 19 & {$[0.95,0.021]$} & {$[19.136,0.021]$} & {$[2.952,-3.85]$} \\
\hline 20 & {$[0.605,0.723]$} & {$[1.531,2.606]$} & {$[0.426,0.958]$} \\
\hline 21 & {$[0.277,0.02]$} & {$[0.383,0.02]$} & {$[-0.96,-3.89]$} \\
\hline 22 & {$[0.954,0.853]$} & {$[20.717,5.789]$} & {$[3.031,1.756]$} \\
\hline
\end{tabular}


Table 6: Continued.

\begin{tabular}{lccc}
\hline Sr. no. & $B(k)$ & $z(k)$ & $\ln \left(Z_{k_{N}}\right)$ \\
\hline 23 & {$[0.875,0.97]$} & {$[7.015,32.67]$} & {$[1.948,3.486]$} \\
24 & {$[0.577,0.876]$} & {$[1.364,7.06]$} & {$[0.311,1.954]$} \\
25 & {$[0.808,0.952]$} & {$[4.216,19.804]$} & {$[1.439,2.986]$} \\
26 & {$[0.902,0.995]$} & {$[9.252,206.617]$} & {$[2.225,5.331]$} \\
27 & {$[0.535,0.645]$} & {$[1.15,1.816]$} & {$[0.14,0.597]$} \\
28 & {$[0.44,0.221]$} & {$[0.786,0.283]$} & {$[3.24,-1.262]$} \\
29 & {$[0.965,0.999]$} & {$[27.261,933.686]$} & $6.839]$ \\
30 & {$[0.532,0.992]$} & {$[0.74,1913,131.102]$} & {$[0.126,4.876]$} \\
31 & {$[0.425,0.999]$} & {$[1.450,4.517]$} & {$[-0.301,7.557]$} \\
32 & {$[0.592,0.819]$} & {$[193.48,43.177]$} & {$[0.371,1.508]$} \\
33 & {$[0.995,0.977]$} & {$[0.838,0.919]$} & {$[-0.265,3.765]$} \\
34 & {$[0.456,0.479]$} & {$[2.159,30.231]$} & {$[0.719,7.854]$} \\
35 & {$[0.672,1.000]$} & {$[1.738,0.404]$} & {$[0.770,3.409]$} \\
36 & {$[0.683,0.968]$} & {$[0.559,111.696]$} & {$[0.553,-0.907]$} \\
37 & {$[0.635,0.288]$} & {$[12.662,13.193]$} & {$[-0.582,4.716]$} \\
38 & {$[0.358,0.991]$} & {$[1.733,5.526]$} & {$[2.539,2.580]$} \\
39 & {$[0.927,0.930]$} & & $1.710]$ \\
40 & {$[0.634,0.847]$} & & {$[0.550,1.75]$} \\
\hline
\end{tabular}

TABle 7: The simulated data for the existing chart.

\begin{tabular}{|c|c|c|c|}
\hline Sr. no.\# & $B(k)$ & $z(k)$ & $\ln \left(Z_{k_{N}}\right)$ \\
\hline 1 & {$[0.352,0.515]$} & {$[0.543,1.063]$} & {$[-0.61,0.061]$} \\
\hline 2 & {$[0.237,0.825]$} & {$[0.31,4.718]$} & {$[-1.171,1.551]$} \\
\hline 3 & {$[0.25,0.424]$} & {$[0.334,0.735]$} & {$[-1.096,-0.308]$} \\
\hline 4 & {$[0.767,0.87]$} & {$[3.291,6.721]$} & {$[1.191,1.905]$} \\
\hline 5 & {$[0.569,0.042]$} & {$[1.322,0.044]$} & {$[0.279,-3.125]$} \\
\hline 6 & {$[0.877,0.019]$} & {$[7.107,0.019]$} & {$[1.961,-3.939]$} \\
\hline 7 & {$[0.359,0.834]$} & {$[0.56,5.039]$} & {$[-0.58,1.617]$} \\
\hline 8 & {$[0.806,0.968]$} & {$[4.153,29.856]$} & {$[1.424,3.396]$} \\
\hline 9 & {$[0.02,0.881]$} & {$[0.021,7.412]$} & {$[-3.88,2.003]$} \\
\hline 10 & {$[0.662,0.071]$} & {$[1.958,0.077]$} & {$[0.672,-2.57]$} \\
\hline 11 & {$[0.909,0.963]$} & {$[9.933,26.211]$} & {$[2.296,3.266]$} \\
\hline 12 & {$[0.959,0.142]$} & {$[23.368,0.166]$} & {$[3.151,-1.797]$} \\
\hline 13 & {$[0.232,0.99]$} & {$[0.302,95.866]$} & {$[-1.199,4.563]$} \\
\hline 14 & {$[0.063,0.965]$} & {$[0.067,27.253]$} & {$[-2.701,3.305]$} \\
\hline 15 & {$[0.918,0.146]$} & {$[11.154,0.172]$} & {$[2.412,-1.763]$} \\
\hline 16 & {$[0.086,0.109]$} & {$[0.094,0.122]$} & {$[-2.364,-2.105]$} \\
\hline 17 & {$[0.936,0.944]$} & {$[14.55,16.968]$} & {$[2.678,2.831]$} \\
\hline 18 & {$[0.198,0.265]$} & {$[0.248,0.36]$} & {$[-1.396,-1.02]$} \\
\hline 19 & {$[0.214,0.605]$} & {$[0.271,1.533]$} & {$[-1.304,0.427]$} \\
\hline 20 & {$[0.507,0.411]$} & {$[1.028,0.699]$} & {$[0.027,-0.359]$} \\
\hline 21 & {$[0.98,0.998]$} & {$[49.554,414.687]$} & {$[3.903,6.028]$} \\
\hline 22 & {$[0.984,0.278]$} & {$[60.027,0.385]$} & {$[4.095,-0.954]$} \\
\hline 23 & {$[0.752,0.979]$} & {$[3.034,46.245]$} & {$[1.11,3.834]$} \\
\hline 24 & {$[0.857,0.713]$} & {$[5.976,2.482]$} & {$[1.788,0.909]$} \\
\hline 25 & {$[0.267,0.946]$} & {$[0.364,17.361]$} & {$[-1.01,2.854]$} \\
\hline 26 & {$[0.744,0.166]$} & {$[2.904,0.199]$} & {$[1.066,-1.614]$} \\
\hline 27 & {$[0.577,0.974]$} & {$[1.363,36.773]$} & {$[0.31,3.605]$} \\
\hline 28 & {$[0.79,0.589]$} & {$[3.761,1.432]$} & {$[1.325,0.359]$} \\
\hline 29 & {$[0.841,0.657]$} & {$[5.275,1.914]$} & {$[1.663,0.649]$} \\
\hline 30 & {$[0.969,0.808]$} & {$[31.497,4.206]$} & {$[3.45,1.436]$} \\
\hline 31 & {$[0.557,0.959]$} & {$[1.257,23.411]$} & {$[0.229,3.153]$} \\
\hline 32 & {$[0.763,0.998]$} & {$[3.228,572.344]$} & {$[1.172,6.35]$} \\
\hline 33 & {$[0.94,0.998]$} & {$[15.578,631.372]$} & {$[2.746,6.448]$} \\
\hline 34 & {$[0.165,0.848]$} & {$[0.197,5.575]$} & {$[-1.625,1.718]$} \\
\hline
\end{tabular}


TABLE 7: Continued.

\begin{tabular}{lccc}
\hline Sr. no.\# & $B(k)$ & $z(k)$ & $\ln \left(Z_{k_{N}}\right)$ \\
\hline 35 & {$[0.873,0.451]$} & {$[6.898,0.822]$} & {$[1.931,-0.196]$} \\
36 & {$[0.836,0.688]$} & {$[5.091,2.207]$} & {$[1.628,0.792]$} \\
37 & {$[0.983,0.928]$} & {$[56.62,12.949]$} & {$[4.036,2.561]$} \\
38 & {$[0.783,0.956]$} & {$[3.61,21.757]$} & {$[1.284,3.08]$} \\
39 & {$[0.631,0.883]$} & {$[1.712,7.528]$} & {$[0.538,2.019]$} \\
40 & {$[0.361,0.998]$} & {$[0.565,488.171]$} & {$[-0.571,6.191]$} \\
\hline
\end{tabular}

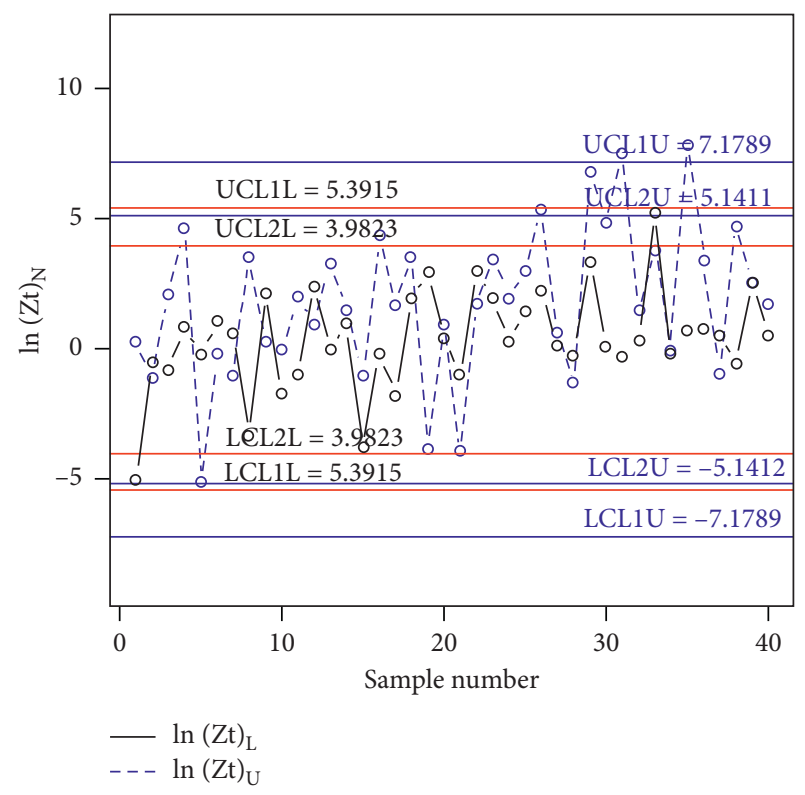

Figure 1: The proposed control chart using simulated data.

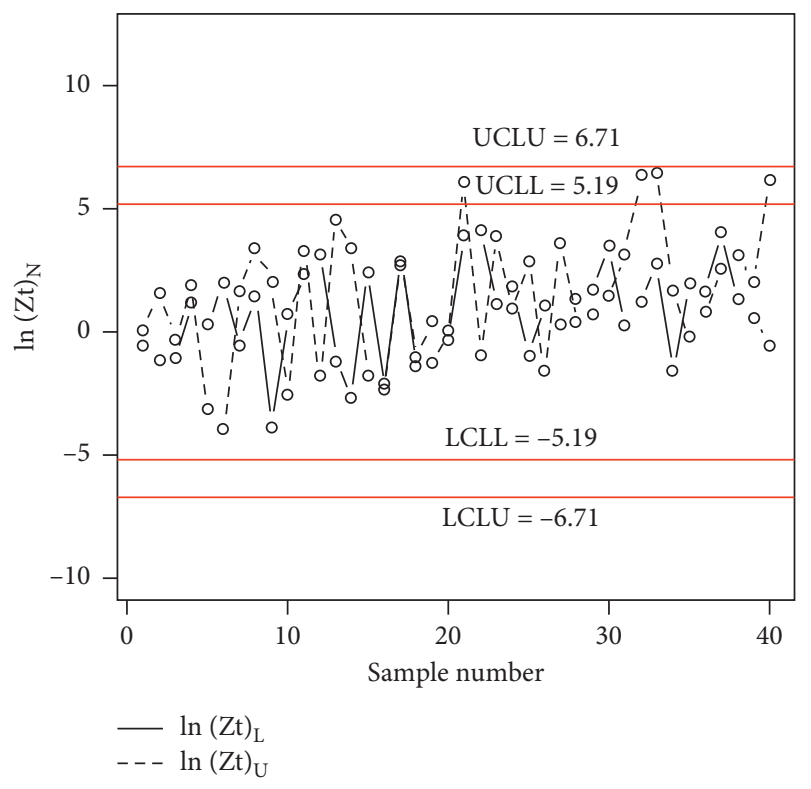

Figure 2: The existing chart proposed by Aslam et al. [44].

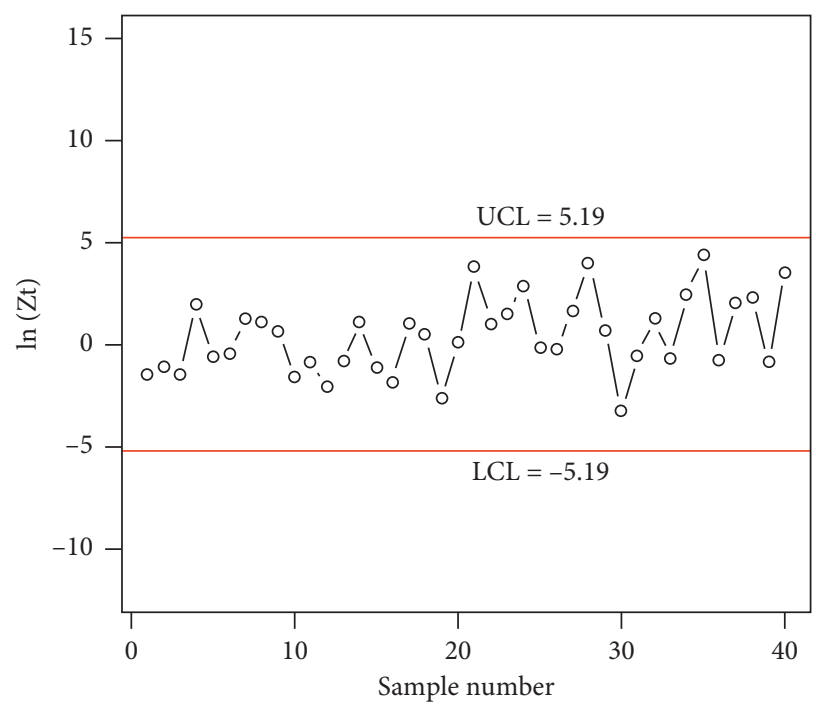

Figure 3: The existing Shewhart chart under classical statistics.

from a big hospital. According to Santiago and Smith [50], "the hospital would like to track the frequency of patients being discharged who had acquired a UTI while in the hospital as a way to quickly identify an increase in infection rate or, conversely, monitor whether forthcoming process or material changes result in fewer infections. Because the root cause often differs based on gender, male and female patients are charted separately and this example focuses on males." Aslam et al. [44] presented the neutrosophic form of UTI data which is shown in Table 8. Suppose that $k_{N}=[3,5], \quad a_{N}=[1.95,2.05], \quad$ and $b_{N}=[2,2.2]$. The four control limits for the UTI data are given as follows:

$$
\begin{aligned}
& \mathrm{UCL1}_{N}=L_{1 N} \sqrt{k_{N}}=[5.3915,7.1789], \\
& \mathrm{LCL1}_{N}=-L_{1 N} \sqrt{k_{N}}=[-5.3915,-7.1789], \\
& \mathrm{UCL} 2_{N}=L_{2 N} \sqrt{k_{N}}=[3.9823,5.1412], \\
& \operatorname{LCL}_{N}=-L_{2 N} \sqrt{k_{N}}=[-3.9823,-5.1412] .
\end{aligned}
$$


TABle 8: The UTI data.

\begin{tabular}{lccc}
\hline Sr. no.\# & $B(k)$ & $z(k)$ & $\ln \left(Z_{k_{N}}\right)$ \\
\hline 1 & {$[0.712,0.448]$} & {$[2.471,0.813]$} & {$[0.905,-0.207]$} \\
2 & {$[0.201,0.89]$} & {$[0.251,8.101]$} & {$[-1.382,2.092]$} \\
3 & {$[0.099,0.897]$} & {$[0.11,8.668]$} & {$[-2.211,2.16]$} \\
4 & {$[0.245,0.087]$} & {$[0.325,0.095]$} & {$[-1.123,-2.354]$} \\
5 & {$[0.417,0.655]$} & {$[0.715,1.896]$} & {$[-0.335,0.64]$} \\
6 & {$[0.083,0.033]$} & {$[0.09,0.034]$} & {$[-2.405,-3.373]$} \\
7 & {$[0.555,0.397]$} & {$[1.245,0.658]$} & {$[0.219,-0.419]$} \\
8 & {$[0.719,0.114]$} & {$[2.555,0.129]$} & {$[0.938,-2.051]$} \\
9 & {$[0.893,0.915]$} & {$[8.361,10.723]$} & {$[2.124,2.372]$} \\
10 & {$[0.697,0.097]$} & {$[2.296,0.108]$} & {$[0.831,-2.23]$} \\
11 & {$[0.573,0.193]$} & {$[1.341,0.239]$} & {$[0.294,-1.432]$} \\
12 & {$[0.234,0.701]$} & {$[0.305,2.346]$} & {$[-1.187,0.853]$} \\
13 & {$[0.932,0.072]$} & {$[13.773,0.078]$} & {$[2.623,-2.55]$} \\
14 & {$[0.533,0.165]$} & {$[1.143,0.197]$} & {$[0.133,-1.622]$} \\
15 & {$[0.134,0.015]$} & {$[0.155,0.016]$} & {$[-1.867,-4.157]$} \\
16 & {$[0.674,0.955]$} & {$[2.071,21.464]$} & {$[0.728,3.066]$} \\
17 & {$[0.008,0.563]$} & {$[0.008,1.286]$} & {$[-4.799,0.251]$} \\
18 & {$[0.241,0.939]$} & {$[0.317,15.27]$} & {$[-1.149,2.726]$} \\
19 & {$[0.481,0.123]$} & {$[0.928,0.14]$} & {$[-0.074,-1.967]$} \\
20 & {$[0.044,0.433]$} & {$[0.046,0.764]$} & {$[-3.071,-0.269]$} \\
21 & {$[0.109,0.928]$} & {$[0.123,12.969]$} & {$[-2.098,2.563]$} \\
22 & {$[0.56,0.236]$} & {$[1.275,0.308]$} & {$[0.243,-1.176]$} \\
23 & {$[0.025,0.844]$} & {$[0.026,5.411]$} & {$[-3.666,1.688]$} \\
24 & {$[0.027,0.153]$} & {$[0.028,0.181]$} & {$[-3.574,-1.71]$} \\
25 & {$[0.826,0.59]$} & {$[4.739,1.438]$} & {$[1.556,0.363]$} \\
26 & {$[0.125,0.872]$} & {$[0.143,6.795]$} & {$[-1.942,1.916]$} \\
27 & {$[0.011,0.361]$} & {$[0.011,0.565]$} & {$[-4.488,-0.571]$} \\
28 & {$[0.025,0.198]$} & {$[0.026,0.247]$} & {$[-3.667,-1.399]$} \\
29 & {$[0.567,0.69]$} & {$[1.311,2.23]$} & {$[0.271,0.802]$} \\
30 & {$[0.909,0.175]$} & {$[9.999,0.211]$} & {$[2.303,-1.554]$} \\
31 & {$[0.325,0.8]$} & {$[0.482,4.006]$} & {$[-0.731,1.388]$} \\
32 & {$[0.126,0.998]$} & {$[0.144,519.999]$} & {$[-1.941,6.254]$} \\
33 & {$[0.474,0.728]$} & {$[0.901,2.671]$} & {$[-0.104,0.982]$} \\
34 & {$[0.35,0.022]$} & {$[0.539,0.022]$} & {$[-0.619,-3.809]$} \\
35 & {$[0.425,0.049]$} & {$[0.74,0.051]$} & {$[-0.301,-2.971]$} \\
36 & {$[0.876,0.723]$} & {$[7.063,2.614]$} & {$[1.955,0.961]$} \\
37 & {$[0.177,0.068]$} & {$[0.215,0.072]$} & {$[-1.539,-2.625]$} \\
38 & {$[0.371,0.99]$} & {$[0.591,100.78]$} & {$[-0.526,4.613]$} \\
39 & {$[0.043,0.173]$} & {$[0.045,0.209]$} & {$[-3.108,-1.565]$} \\
40 & {$[0.117,0.191]$} & {$[0.132,0.235]$} & {$[-2.022,-1.446]$} \\
\hline & & &
\end{tabular}

Using the given information, the value of $Z_{k_{N}}$ when $Z_{0}=1$ and $B\left(O_{0}\right)=0.5$ is calculated as follows:

$$
Z_{k_{N}}=Z_{k_{N}-1} e^{T_{N}^{*}-\mu_{T_{N}^{*}} / \sigma_{T_{N}^{*}}}=[0.985,54.568] .
$$

The neutrosophic statistic $\ln \left(Z_{k_{N}}\right)$ is plotted on four control limits in Figure 4. The planned control chart shows the out-of-control process after the $6^{\text {th }}$ sample. The measures of $\ln \left(Z_{k_{N}}\right)$ are also plotted in Figure 5 using the chart of neutrosophic statistics proposed by Aslam et al. [44] which shows the out-of-control process at the $32^{\text {nd }}$ sample. The same data were also plotted in Figure 6 using

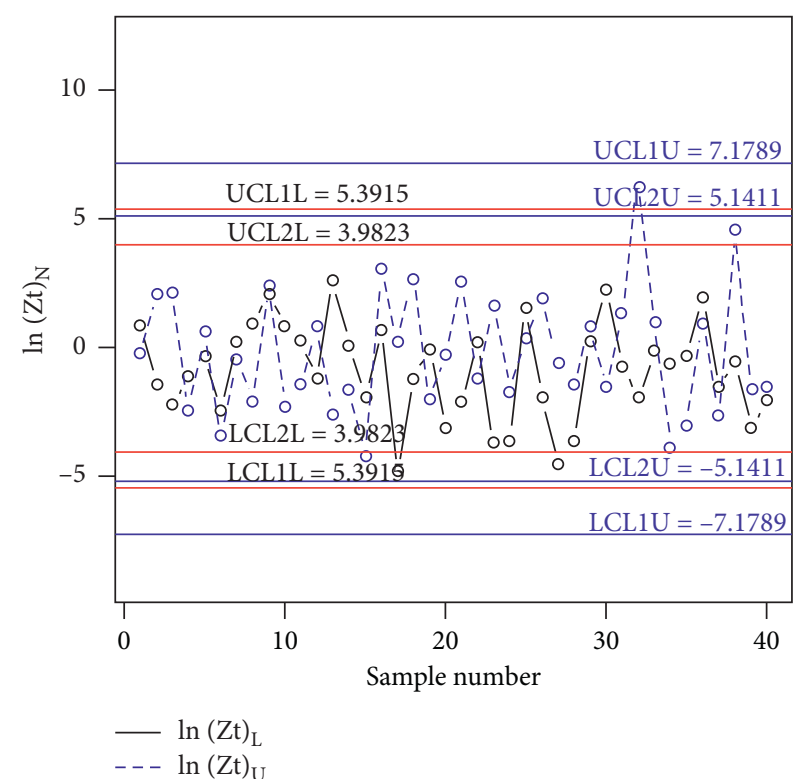

FIgURE 4: The proposed chart for UTI data.

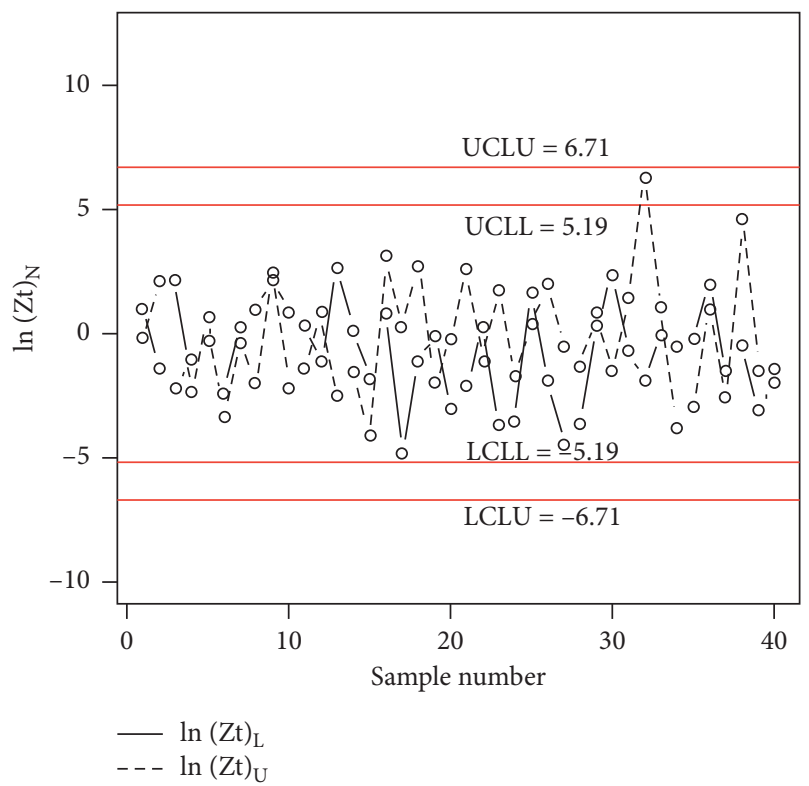

Figure 5: Aslam et al. [44] chart for UTI data.

the Shewhart chart which is incapable to identify the outof-control situation. Therefore, the planned chart is efficient in identifying the out-of-control situation more swiftly. The use of the proposed control chart in the hospital will help to identify the UTI patient quickly as compared to the existing charts. 


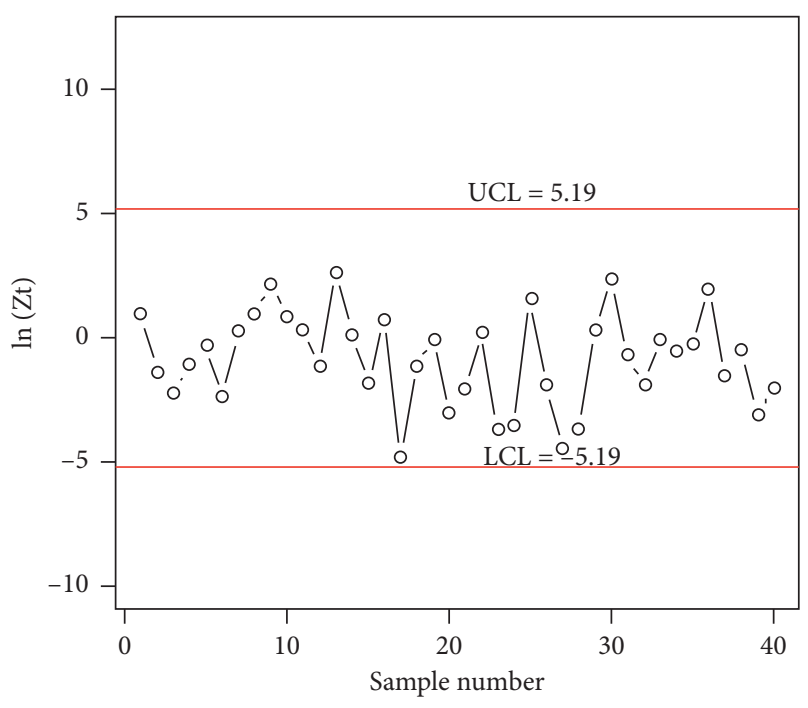

Figure 6: Shewhart chart for UTI data.

\section{Conclusions}

In this article, the planning of a control chart for gammadistributed belief statistic using MDS sampling under the neutrosophic statistic has been offered. The parameters of the planned chart have been estimated for vague data using code programming for $\mathrm{R}$ language. Neutrosophic average run lengths for indeterminacy intervals under different process settings for various shift levels have been calculated. The comparison of the planned scheme with the existing chart has been made which shows the better identifying skill of the outof-control process. It has been perceived that the proposed scheme is a valuable accumulation in the toolkit of the quality control professionals for the monitoring of neutrosophic data. A real-world example has been added for the practical application of the planned scheme by the quality control workers. The proposed chart ensures the producer/customer that the product manufactured using the proposed control chart will be according to the given specification limits and good quality product as mentioned in ISO 9001: 2015 Quality Management Systems (https://www.iso.org). The proposed chart can be applied in the industry to minimize the nonconforming product. The proposed chart has the limitation that it can be applied when the quality of interest follows the normal distribution. The proposed control chart for nonnormal distributions can be considered as future research. The planned chart can further be extended for the multivariate probability distributions. The proposed control chart using the cost model can be studied as future research.

\section{Data Availability}

The data used to support the findings of this study are included within the article.

\section{Conflicts of Interest}

The authors declare that they have no conflicts of interest.

\section{Acknowledgments}

This work was funded by the Deanship of Scientific Research (DSR), King Abdulaziz University, Jeddah, under grant no. (G-1407-130-1440). The authors, therefore, gratefully acknowledge the DSR technical and financial support.

\section{References}

[1] D. K. Bhaumik and R. D. Gibbons, "One-sided approximate prediction intervals for at leastpofm observations from a gamma population at Each Of relocations," Technometrics, vol. 48 , no. 1 , pp. 112-119, 2006.

[2] M. Aslam, O.-H. Arif, and C.-H. Jun, "A control chart for gamma distribution using multiple dependent state sampling," Industrial Engineering and Management Systems, vol. 16, no. 1, pp. 109-117, 2017.

[3] M. Aslam, N. Khan, and C.-H. Jun, "A control chart using belief information for a gamma distribution," Operations Research and Decisions, vol. 26, no. 4, pp. 5-19, 2016.

[4] C. W. Zhang, M. Xie, J. Y. Liu, and T. N. Goh, "A control chart for the gamma distribution as a model of time between events," International Journal of Production Research, vol. 45, no. 23, pp. 5649-5666, 2007.

[5] H. A. Al-Oraini and M. A. Rahim, "Economic statistical design of control charts for systems with gamma $(\lambda, 2)$ incontrol times," Computers \& Industrial Engineering, vol. 43, no. 3, pp. 645-654, 2002.

[6] H. Aksoy, "Use of gamma distribution in hydrological analysis," Turkish Journal of Engineering and Environmental Sciences, vol. 24, no. 6, pp. 419-428, 2000.

[7] B. Bobée and F. Ashkar, The Gamma Family and Derived Distributions Applied Inhydrology, Water Resources Publications, Fort Collins, CO, USA, 1991.

[8] I. M. Gonzalez and E. Viles, "Design of $\mathrm{r}$ control chart assuming a gamma distribution," Economic Quality Control, vol. 16, no. 2, pp. 199-204, 2001.

[9] A. W. Wortham and R. C. Baker, "Multiple deferred state sampling inspection," International Journal of Production Research, vol. 14, no. 6, pp. 719-731, 1976.

[10] V. Soundararajan and R. Vijayaraghavan, "On designing multiple deferred state sampling (MDS-1 $(0,2)$ plans involving minimum risks," Journal of Applied Statistics, vol. 16, no. 1, pp. 87-94, 1989.

[11] V. Soundararajan and R. Vijayaraghavan, "Construction and selection of multiple dependent (deferred) state sampling plan," Journal of Applied Statistics, vol. 17, no. 3, pp. 397-409, 1990.

[12] V. Kuralmani and K. Govindaraju, "Selection of multiple deferred (dependent) state sampling plans," Communications in Statistics-Theory and Methods, vol. 21, no. 5, pp. 13391366, 1992.

[13] K. Govindaraju and K. Subramani, "Selection of multiple deferred (dependent) state sampling plans for given acceptable quality level and limiting quality level," Journal of Applied Statistics, vol. 20, no. 3, pp. 423-428, 1993.

[14] S. Balamurali and C.-H. Jun, "Multiple dependent state sampling plans for lot acceptance based on measurement data," European Journal of Operational Research, vol. 180, no. 3, pp. 1221-1230, 2007.

[15] M. Aslam, N. Khan, and C.-H. Jun, "A multiple dependent state control chart based on double control limits," Research Journal of Applied Sciences, Engineering and Technology, vol. 7, no. 21, pp. 4490-4493, 2014. 
[16] M. Aslam, C.-H. Yen, C.-H. Chang, and C.-H. Jun, "Multiple dependent state variable sampling plans with process loss consideration," The International Journal of Advanced Manufacturing Technology, vol. 71, no. 5-8, pp. 1337-1343, 2014.

[17] M. Aslam, M. Azam, N. Khan, and C.-H. Jun, "A control chart for an exponential distribution using multiple dependent state sampling," Quality \& Quantity, vol. 49, no. 2, pp. 455-462, 2015.

[18] M. Aslam, A. Nazir, and C.-H. Jun, "A new attribute control chart using multiple dependent state sampling," Transactions of the Institute of Measurement and Control, vol. 37, no. 4, pp. 569-576, 2015.

[19] S. Balamurali, P. Jeyadurga, and M. Usha, "Designing of bayesian multiple deferred state sampling plan based on gamma-poisson distribution," American Journal of Mathematical and Management Sciences, vol. 35, no. 1, pp. 77-90, 2016.

[20] A. Yan, S. Liu, and X. Dong, "Designing a multiple dependent state sampling plan based on the coefficient of variation," SpringerPlus, vol. 5, no. 1, p. 1447, 2016.

[21] M. S. Aldosari, M. Aslam, and C.-H. Jun, "A new attribute control chart using multiple dependent state repetitive sampling," IEEE Access, vol. 5, 2017.

[22] W. Zhou, Q. Wan, Y. Zheng, and Y.-W. Zhou, “A jointadaptive np control chart with multiple dependent state sampling scheme," Communications in Statistics-Theory and Methods, vol. 46, no. 14, pp. 6967-6979, 2017.

[23] R. Afshari and B. Sadeghpour Gildeh, "Designing a multiple deferred state attribute sampling plan in a fuzzy environment," American Journal of Mathematical and Management Sciences, vol. 36, no. 4, pp. 328-345, 2017.

[24] J. Chen, J. Ye, and S. Du, "Scale effect and anisotropy analyzed for neutrosophic numbers of rock joint roughness coefficient based on neutrosophic statistics," Symmetry, vol. 9, no. 10, p. 208, 2017.

[25] F. Smarandache, Neutrosophy, Neutrosophic Probability, Set and Logic, Latern Press, Seattle, WA, USA, 1998.

[26] F. Smarandache, "Introduction to neutrosophic statistics: infinite study," 2014, https://arxiv.org/ftp/arxiv/papers/1406/ 1406.2000.pdf.

[27] J. Ye, J. Chen, R. Yong, and S. Du, "Expression and analysis of joint roughness coefficient using neutrosophic number functions," Information, vol. 8, no. 2, p. 69, 2017.

[28] M. Aslam, "A new sampling plan using neutrosophic process loss consideration," Symmetry, vol. 10, no. 5, p. 132, 2018.

[29] M. Aslam and A. Al-Marshadi, "Design of sampling plan using regression estimator under indeterminacy," Symmetry, vol. 10, no. 12, p. 754, 2018.

[30] M. Aslam and O. Arif, "Testing of grouped product for the weibull distribution using neutrosophic statistics," Symmetry, vol. 10, no. 9, p. 403, 2018.

[31] M. Aslam, N. Khan, and M. Albassam, "Control chart for failure-censored reliability tests under uncertainty environment," Symmetry, vol. 10, no. 12, p. 690, 2018.

[32] M. Aslam, N. Khan, and M. Khan, "Monitoring the variability in the process using neutrosophic statistical interval method," Symmetry, vol. 10, no. 11, p. 562, 2018.

[33] M. Aslam, R. A. R. Bantan, and N. Khan, "Design of a new attribute control chart under neutrosophic statistics," International Journal of Fuzzy Systems, vol. 21, no. 2, pp. 433-440, 2019.

[34] R. Jansi, K. Mohana, and F. Smarandache, "Correlation measure for pythagorean neutrosophic sets with $t$ and $f$ as dependent neutrosophic components," Neutrosophic Sets \& Systems, vol. 30, 2019.

[35] P. Muralikrishna and D. S. Kumar, "Neutrosophic Approach on Normed Linear Space," Neutrosophic Sets \& Systems, vol. 30, 2019.

[36] F. Amin, A. Fahmi, and M. Aslam, "Approaches to multiple attribute group decision making based on triangular cubic linguistic uncertain fuzzy aggregation operators," Soft Computing, vol. 24, no. 15, 2019.

[37] S. Dobbah, M. Aslam, and K. Khan, "Design of a new synthetic acceptance sampling plan," Symmetry, vol. 10, no. 11, p. 653, 2018.

[38] S. Islam and S. C. Deb, "Neutrosophic goal programming approach to a green supplier selection model with quantity discount," Neutrosophic Sets \& Systems, vol. 30, 2019.

[39] M. Kashif, H. Nida, M. I. Khan, and M. Aslam, "Decomposition of matrix under neutrosophic environment," $\mathrm{Neu}$ trosophic Sets \& Systems, vol. 30, 2019.

[40] M. Mashuri and M. Ahsan, "Perfomance fuzzy multinomial control chart," Journal of Physics: Conference Series, vol. 1028, 2018.

[41] X. Peng and J. Dai, "Approaches to single-valued neutrosophic madm based on mabac, topsis and new similarity measure with score function," Neural Computing and Applications, vol. 29, no. 10, pp. 939-954, 2018.

[42] M.-H. Shu, D.-C. Dang, T.-L. Nguyen, B.-M. Hsu, and N.-S. Phan, "Fuzzy and control charts: a data-adaptability and human-acceptance approach," Complexity, vol. 2017, Article ID 4376809, 17 pages, 2017.

[43] C. M. Villamar, J. Suarez, L. De Lucas Coloma, C. Vera, and M. Leyva, "Analysis of technological innovation contribution to gross domestic product based on neutrosophic cognitive maps and neutrosophic numbers," Neutrosophic Sets \& Systems, vol. 30, 2019.

[44] M. Aslam, R. A. R. Bantan, and N. Khan, "Monitoring the process based on belief statistic for neutrosophic gamma distributed product," Processes, vol. 7, no. 4, p. 209, 2019.

[45] E. B. Wilson and M. M. Hilferty, "The distribution of chisquare," Proceedings of the National Academy of Sciences, vol. 17, no. 12, pp. 684-688, 1931.

[46] M. S. Fallah Nezhad and S. T. Akhavan Niaki, "A new monitoring design for uni-variate statistical quality control charts," Information Sciences, vol. 180, no. 6, pp. 1051-1059, 2010.

[47] C. D. Montgomery, Introduction to Statistical Quality Control, John Wiley \& Sons, New York City, NY, USA, 2009.

[48] L. Ahmad, M. Aslam, and C.-H. Jun, "Coal quality monitoring with improved control charts," European Journal of Scientific Research, vol. 125, no. 2, pp. 427-434, 2014.

[49] L. Ahmad, M. Aslam, and C.-H. Jun, "Designing of x-bar control charts based on process capability index using repetitive sampling," Transactions of the Institute of Measurement and Control, vol. 36, no. 3, pp. 367-374, 2014.

[50] E. Santiago and J. Smith, "Control charts based on the exponential distribution: adapting runs rules for the t chart," Quality Engineering, vol. 25, no. 2, pp. 85-96, 2013. 